Urs Jäger

Wertbewusstes Controlling 
Urs Jäger

\section{Wertbewusstes Controlling}

Harte und weiche Faktoren integrieren 
Bibliografische Information Der Deutschen Bibliothek

Die Deutsche Bibliothek verzeichnet diese Publikation in der Deutschen Nationalbibliografie; detaillierte bibliografische Daten sind im Internet über <http://dnb.ddb.de> abrufbar.

1. Auflage 2003

Alle Rechte vorbehalten

(c) Betriebswirtschaftlicher Verlag Dr. Th. Gabler/GWV Fachverlage GmbH, Wiesbaden 2003

Lektorat: Ulrike M. Vetter

Der Gabler Verlag ist ein Unternehmen der Fachverlagsgruppe BertelsmannSpringer. www.gabler.de

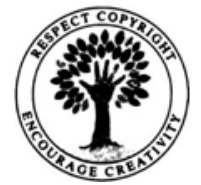

Das Werk einschließlich aller seiner Teile ist urheberrechtlich geschützt. Jede Verwertung außerhalb der engen Grenzen des Urheberrechtsgesetzes ist ohne Zustimmung des Verlags unzulässig und strafbar. Das gilt insbesondere für Vervielfältigungen, Übersetzungen, Mikroverfilmungen und die Einspeicherung und Verarbeitung in elektronischen Systemen.

Die Wiedergabe von Gebrauchsnamen, Handelsnamen, Warenbezeichnungen usw. in diesem Werk berechtigt auch ohne besondere Kennzeichnung nicht zu der Annahme, dass solche Namen im Sinne der Warenzeichen- und Markenschutz-Gesetzgebung als frei zu betrachten wären und daher von jedermann benutzt werden dürften.

Umschlaggestaltung: Nina Faber de.sign, Wiesbaden

Gedruckt auf säurefreiem und chlorfrei gebleichtem Papier 


\section{Vorwort}

Jeder Controller, der es einmal versuchte, weiß, wie schwer es ist, neutral und objektiv zu sein. Es ist schwer, um nicht zu sagen unmöglich, weil das Controlling Zahlen, Daten und Fakten aus Sicht einer bestimmten Werthaltung interpretiert und Einfluss auf seine Organisation nimmt. Das Controlling kann sich nur bemühen, eine neutrale Position in sachlichen und politischen Auseinandersetzungen einzunehmen, und es kann nur versuchen, sachgerecht zu bleiben, weil es keine objektive Bewertungen erarbeitet. Controlling wirkt zudem genauso auf seine Organisation ein und wird von ihr beeinflusst wie jede Management-Funktion, weshalb es seine Berichte und Analysen nicht als unabhängige Funktion kommuniziert.

Auf Basis solcher Beobachtungen schlägt dieses Buch den Ansatz „wertbewusstes Controlling“ vor. Dieser Vorschlag versucht, einen Weg zu begründen, wie Zahlen, Daten und Fakten interpretiert und wie "harte" und „weiche“ Faktoren bewusst und systematisch in Controlling-Prozessen reflektiert und integriert werden können. Eine solche Methode ist umso dringender, je stärker sich Controlling in einer Welt zurechtfinden muss, in der „weiche“ Faktoren ökonomische Erfolge mitbestimmen.

Dieser Versuch, ein „wertbewusstes Controlling“ zu begründen, hat seinen Ursprung in Praxiserfahrungen. Zweieinhalb Jahre war ich bei der Deutschen Lufthansa AG als Controller tätig. Während dieser Zeit führte ich zahlreiche Gespräche mit Controllern aus den unterschiedlichsten Abteilungen und Bereichen. Ich war überrascht, auf wenige Controller zu treffen, die überzeugt waren, ihre Organisation verstehen zu können, und glaubten, über allgemeingültige Rezepte der Problemlösung zu verfügen. Die Meisten stellten in Bezug auf ihre Organisation kompetent eine Frage nach der anderen. Es scheint, als ob Controlling-Erfahrung zu Bescheidenheit und zum Bewusstsein der eigenen beschränkten Möglichkeiten führt.

Ich war zudem erstaunt, was die Gesprächspartner auf die Frage antworteten, nach welchen Methoden sie arbeiten würden: Die meisten berichteten von zahlenorientierten Controlling-Instrumenten. Auf die Nachfrage, was sie an einem typischen Arbeitstag täten, beschrieben sie dagegen Tätigkeiten, die weit über die zahlenorientierten Instrumente hinausgehen und weit in Themenfelder des General-Managements hineinreichen. Die eigentlichen Zahlen und mathematischen Controlling-Methoden rückten in den Hintergrund. Sie wurden als Instrumente dargestellt, die jeder im Controlling wie das Schalten beim Autofahren beherrschen muss. 
Wenn die eigentlichen Controlling-Instrumente in den Hintergrund treten, ist von Interesse, was an ihrer Stelle im Vordergrund steht. Um dies herauszufinden, führte ich Interviews durch und beobachtete mit gesteigerter Aufmerksamkeit mein Tagesgeschäft als Controller und dasjenige meiner Kollegen.

Dieses Buch entstand durch zahlreiche Gespräche und Ratschläge. Besonders danken möchte ich Dr. Heiko Lange, ehemals Konzern-Personalvorstand der Deutschen Lufthansa AG, der das gesamte Buch als konstruktiv-kritischer Gesprächspartner und Leser begleitete. Zahlreiche seiner Hinweise und seiner Ratschläge prägen die Inhalte dieser Arbeit. Ebenso möchte ich Thomas Sattelberger, Bereichsvorstand „Product and Services" der Lufthansa Passage, für seine motivierende Unterstützung und für seine konstruktiven Hinweise danken. Mein Mentor, Harald Deprosse, Leiter „Zentrale Verkehrsleitung und Besatzungsplanung", begleitete diese Arbeit mit seinen Gedanken und war wohl immer einen Schritt voraus. Er prägte dieses Buch wesentlich. Dr. Peter Hach, ehemals Leiter Konzern-Controlling, danke ich für seine konstruktive Kritik, die an zahlreichen Stellen dieser Arbeit einfloss.

Prof. Ekkehard Kappler von der Universität Innsbruck motivierte mich durch seine Hinweise und seine Impulse erst richtig für diese Arbeit. Prof. Johannes Rüegg-Stürm von der Universität St.Gallen gab mir zahlreiche Hinweise, welche die Struktur dieses Buchs bestimmen. Mein Vater, Prof. Alfred Jäger von der kirchlichen Hochschule Bethel, unterstützte mich maßgeblich durch seine ideenreiche Kritik, durch seine visionären Ideen und durch motivierendes Zureden.

Einige erfahrene Praktiker der Lufthansa Passage, Reinhard Schäfer, Leiter Direktvertrieb, Thomas Klühr, Leiter Controlling, Annette Frühauf, Leiterin Vertriebs-Controlling, und Michael Baltes, Vertriebscontroller, unterstützten mich während dieser Arbeit. Sie ermöglichten es, dass dieses Buch auch am Rande einer normalen beruflichen Tätigkeit entstehen konnte, und sie gaben zahlreiche Ratschläge, die sich in vielen Beispielen dieser Arbeit niederschlugen. Susanne Schwarzer, Tanja Eibich, Grit KöhlerSvennson und Thomas Lemper danke ich für die gute Zusammenarbeit während der Durchführung eines Projektes, in dem nach interpretativen Controlling-Methoden gearbeitet wurde.

Ein ganz herzlicher Dank geht an Claudia Reimer und an Michael Jäger, die in zahlreichen Stunden diese Arbeit kompetent und in kurzer Zeit auf ihre Fehler hin prüften und korrigierten, und an den Gabler-Verlag, der diese Arbeit verlegt. Claudia Cristina Hubschmid möchte ich von ganzem Herzen danken für ihre ausgedehnte Geduld und für ihre fachlich-kritische und moralische Unterstützung; ihr ist diese Arbeit gewidmet. 


\section{Inhaltsverzeichnis}

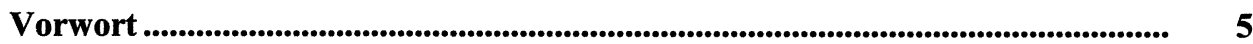

1 Einleitung: Professionalisierung........................................................................ 9

2 Arbeiten nach interpretativen Controlling-Methoden ......................................... 19

2.1 Offenes Denken und Wahrnehmen .............................................................. 20

2.2 „Weiche“ und „harte“ Faktoren erfassen ...................................................... 37

2.3 Erster Impuls: Controlling-Aufgaben dynamisch verstehen.......................... 44

3 Lernen im Management unterstützen................................................................... 53

3.1 Bewusstes Lernen .............................................................................. 57

3.2 Planung als Orientierungsprozess des Lernens ............................................ 82

3.3 Zweiter Impuls: „Kritisches Denken“ im Management fördern..................... 100

4 Interpretation prägt seit jeher das Controlling.................................................... 119

4.1 Weiche Faktoren hart rechnen ............................................................ 121

4.2 Der weiche Kern harter Faktoren ................................................................. 136

4.3 Dritter Impuls: Der Interpretations-Kreislauf ............................................. 148

5 Situationsorientierter Einsatz ............................................................................ 157

5.1 Sachgemäße Moderation......................................................................... 160

5.2 Beziehungsgerechte Moderation.............................................................. 179

5.3 Vierter Impuls: Professionalisierung durch wertbewusstes Controlling ....... 191 


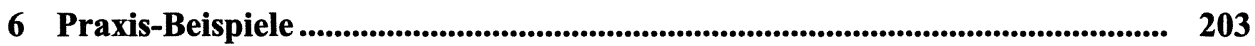

6.1 Balanced Scorecard als Moderationsinstrument ......................................... 203

6.2 Balanced Scorecard in bekannte Instrumente integrieren ............................. 205

6.3 Wenn Controller nach interpretativen Methoden arbeiten ............................. 208

6.4 WAR-Analyse als Basis eines tiefen Organisationsverständnisses.............. 212

6.5 Alltagstheorien durch kritisches Hinterfragen aufdecken ............................. 217

7 Schlussbemerkung: kritisches Vor-Denken........................................................... 227

Literaturverzeichnis .............................................................................................................. 229

Abbildungsverzeichnis ............................................................................................................. $\quad 235$

Der Autor .............................................................................................................................. 239 